\title{
Transmission Power Control in Mobile Wireless Sensor Networks: Simulation-based Approach
}

\author{
Mohamad Nazim Jambli*, Halikul Lenando*, Kartinah Zen*, Sinarwati Mohamad Suhaili ${ }^{\dagger}$, Alan Tully \\ ${ }^{*}$ Faculty of Computer Science \& Information Technology, Universiti Malaysia Sarawak, Malaysia \\ $\dagger$ Pre-University Studies, Universiti Malaysia Sarawak, Malaysia \\ ${ }^{\ddagger}$ School of Computing Science, Newcastle University, UK
}

\begin{abstract}
Saving energy is a very critical issue in wireless sensor networks (WSNs) because sensor nodes have a severe resource constraints such as lack of processing power and limited in power supply. Since the communication is the most energy consuming activities in WSNs, the power use for transmission or reception of packet should be managed properly. Transmission power control (TPC) technique is one of the techniques to reduce energy consumption which has been widely studied in mobile ad-hoc networks (MANETs). This technique is implemented by adjusting the transmission power in communication between nodes. However, as mobile wireless sensor networks (MWSNs) applications emerge, the unique characteristics of this network such as severe resource constraints and frequent topology change suggest that TPC might be useful to reduce energy consumption in MWSN. Therefore, we investigate the impact of TPC on Ad hoc On-Demand Distance Vector (AODV) routing protocol for MWSNs. AODV is used as a medium of communication to assist the investigation of the effects of TPC in multihop communication in MWSNs. The simulation results show that the implementation of TPC technique has some impact on MWSNs in respect to transmission energy consumption and transmission power level required at low node mobility.

Index Terms-MANETs, MWSNs, TPC, AODV, Simulation
\end{abstract}

\section{INTRODUCTION}

In recent years, extensive research has been conducted on Wireless Sensor Networks (WSNs) due to their wide range of potential applications. The enormous potential of this technology can be seen ranging from environmental monitoring to critical military surveillance and healthcare applications [1]. In these networks a large number of small sensor nodes are deployed, each capable of collecting, storing, processing observations and communicating over short-range wireless interfaces and multiple hops to central locations called sinks. However, the nodes in WSNs have severe resource constraints due to their lack of processing power, limited memory, bandwidth and energy [2]. Since these networks are usually deployed in remote places and left unattended, they should be equipped with energy-constraint routing protocol to increase the network lifetime.

The researchers in WSNs have proposed various routing protocols which are optimized for these networks with resource constraints. A number of efficient routing protocols has been proposed by several researchers in WSNs [3], [4]. When describing the existing routing protocols, normally they assumed that sensor nodes and sinks are stationary in the deployment. But for some WSN applications, a mobile node is also present because a mobile Wireless Sensor Network (MWSN) owes its name to the presence of mobile sink or sensor nodes within the network [5]. For example in ocean temperature monitoring application, the sensors are deploy on the surface of the ocean to monitor the water temperature and we can expect that they are carried around by ocean flows [6]. As for a real-time target tracking in battle situations, mobile nodes can be used to avoid holes in the coverage and to generate information to be transmitted through the network [7]. In some other applications, sensors are mounted on robots, animals or other moving objects, which can sense and collect relevant information. If this information is not managed properly, energy can be wasted due to unpredictable changes in network topology in mobility environment.

It is even more difficult to design routing protocol in mobile wireless sensor networks (MWSNs) because when some of the sensor nodes are mobile it is not easy to detect any broken routes and react faster to topology change. The increase of mobility in sensor nodes also affects the connection to their neighbours and the routing table update as evaluated in our previous work in [8]. Moreover, the communication process between sensor nodes when they are moving from one area to another also consumes more energy related to transmitting and receiving control packets. Since the communication is the most energy-consuming activities in WSNs, the power use for transmission or reception of packet should be controlled as much as possible in designing any routing protocol for MWSNs. The adjustment of transmission power through dynamic transmission power control (TPC) protocols is one of the techniques to effectively reduce energy consumption in WSNs [9]. Therefore, in this paper we investigate the impact of TPC technique on MWSNs. AODV routing protocol is used as a medium of communication to assist our investigation using Random Way Point (RWP) mobility model to simulate mobile environment in MWSNs.

The rest of the paper is organized as follows. In Section II, we includes the recent related work on TPC technique. Next, we briefly described transmission power control for MWSNs in Section III. Then, the AODV routing protocol is summarized in section IV. The simulation tool and metrics are outlined in Section V. We present the simulation results in section VI. Section VII concludes the paper and outlines the future work. 